$\underline{\text { Revised }}$

Original Article

\title{
Treatment of central nervous system lymphoma in rats with intraventricular rituximab and serum
}

\author{
Running head: \\ CNSL therapy model with intraventricular rituximab and serum
}

Yasuyuki Miyake*, Yasushi Okoshi*, Takayuki Machino and Shigeru Chiba

(*These authors contributed equally to this work.)

Clinical and Experimental Hematology, Major of Clinical Sciences,

Graduate School of Comprehensive Human Sciences, University of Tsukuba

1-1-1, Tennodai, Tsukuba, Ibaraki 305-8575, Japan.

\author{
Correspondence to \\ Yasushi Okoshi, M.D., Ph.D. (yokoshi@.md.tsukuba.ac.jp) \\ Shigeru Chiba, M.D., PhD. (schiba-tky@umin.net) \\ Tel: +81-29-853-3127, Fax: +81-29-853-8079
}




\section{Abstract}

B-cell lymphomas often develop in the central nervous system (CNS). Although rituximab (RTX) has been widely used for most B-cell lymphomas, the efficacy for CNS lymphomas has yet to be elucidated. A major concern is that RTX might not reach lymphoma lesions, and either the antibody-dependent cellular cytotoxicity or complement-dependent cytotoxicity (CDC) might not substantially operate in the CNS environment. Here we investigated the potential usefulness of co-administering RTX and human serum intraventricularly in nude rats carrying human B-cell lymphomas in the CNS. Raji, a CD20-positive lymphoma cell line, was inoculated into the cerebrum of F344 (rnu/rnu) nude rats. After several days, RTX and human serum were delivered into the ipsilateral lateral ventricle via a cannula. Intraventricularly administered RTX was localized specifically at the lymphoma lesions, indicating that RTX penetrated the ependymal layer of the lateral ventricle to reach the tumor lesion, where it specifically bound to the lymphoma cells. The combination of RTX and serum $(n=12)$, but not RTX alone $(n=13)$, significantly extended the survival of the rats $(P=0.049)$. Intraventricular administration of RTX and serum in a rat/human CNS lymphoma model might be a potential novel treatment for CNS lymphomas of B-cell origin. Clinical trials are warranted.

Keywords: CNS lymphoma, rituximab, complement-dependent cytotoxicity, intraventricular 
Central nervous system (CNS) involvement is one of the serious manifestations of B-cell

lymphomas. It occurs as extranodal involvement of systemic lymphoma, often during relapse, or as a primary CNS lymphoma, which is now termed "primary diffuse large B-cell lymphoma (DLBCL) of the CNS" in version 4 of the WHO classification [1]. High-dose methotrexate with or without radiotherapy is the current mainstay of treatment. However, side effects, such as severe cytopenia, renal insufficiency, and delayed neurological damage are a concern, especially in heavily pretreated or elderly patients. Even if these therapies are initially effective, long-term outcomes remain poor [2-4].

Rituximab (RTX) is a monoclonal antibody against CD20, a four-transmembrane protein whose expression is confined to precursor and mature B cells [5, 6]. RTX, which consists of the variable region of mouse antibody and the constant region of human IgG1 [7], is widely used to treat systemic B-cell lymphomas. RTX is considered to have its therapeutic effect through (i) antibody-dependent cell-mediated cytotoxicity (ADCC), mainly mediated by natural killer cells [8-10]; (ii) complement-dependent cytotoxicity (CDC) mediated by complement factors that are activated by binding of the complement protein C1q to RTX [8, 10-14]; and (iii) induction of tumor cell apoptosis by CD20 stimulation [15-17]. Despite the fact that intravenous RTX is effective against most B-cell lymphomas, its efficacy against B-cell lymphomas developing in the CNS (hereafter designated CNSL) 
has not yet been shown. The presence of the blood-brain barrier might hamper intravenous RTX from

reaching the CNSL, given that the concentration of RTX in the cerebrospinal fluid (CSF) is $1 \%$ or less of that in the blood when it is administered intravenously [18-20].

CSF is produced at the choroid plexus in the lateral ventricle, flows through the third and fourth ventricle, and finally flows into the subarachnoid space. CSF also spreads from the lateral ventricle into the brain parenchyma through ependymal cells, which form a thin epithelial membrane lining the cerebral ventricle [21]. Some cytokines and antibodies administered into the lateral ventricle do penetrate into the brain parenchyma [21-24]. In addition, the pia mater, which directly covers the brain surface, is also a leaky structure [21]. Therefore, intrathecal or intraventricular administration of RTX could be a viable choice of treatment for CNSL. However, another important concern about the potential effectiveness of RTX against CNSL is that the CNS environment lacks immune cells and complement, the two key factors needed for RTX to kill the target cells.

To investigate whether RTX can be used to treat CNSL, we developed a human CNSL xenograft model in rats and evaluated the efficacy of intraventricular co-administration of RTX and human serum. 
2 Materials and methods

\subsection{Antibodies and serum}

RTX was kindly supplied by Zenyaku Kogyo Co. Ltd (Tokyo, Japan). Human polyclonal immunoglobulin $\left(\right.$ Gammagard $^{\circledR}$, Baxter, Deerfield, IL) was used as a control immunoglobulin for RTX. Human serum was separated from the clotted whole blood of a healthy volunteer by centrifugation at $1600 \times g$ and stored at $-30^{\circ} \mathrm{C}$. Inactivated serum was prepared by placing the human serum at $56^{\circ} \mathrm{C}$ for 30 minutes.

\subsection{Cell line and cell culture}

Raji, a CD20-positive human Burkitt lymphoma cell line, was obtained from RIKEN BioResource Center (Tsukuba, Japan) and was cultured in RPMI1640 medium (Sigma-Aldrich, St. Louis, MO) supplemented with $10 \%$ fetal bovine serum (Sigma-Aldrich) and penicillin and streptomycin (Sigma-Aldrich; used at 100 units $/ \mathrm{mL}$ and $100 \mu \mathrm{g} / \mathrm{mL}$, respectively) at $37^{\circ} \mathrm{C}$ with $10 \%$ $\mathrm{CO}_{2}$ in humidified air.

\subsection{Complement-dependent cytotoxicity assay}

Raji cells $\left(1.0 \times 10^{5}\right)$ were cultured in medium containing $20 \mu \mathrm{g} / \mathrm{mL} \mathrm{RTX}$ and various 
concentrations of human serum in 96-well round-bottom dishes for 7 hours. After the incubation, $50 \mu \mathrm{L}$

of propidium iodide (PI; $7.5 \mu \mathrm{g} / \mathrm{mL}$ ) was added and analyzed by flow cytometry (FACSCalibur ${ }^{\circledR}$, Becton

Dickinson, Franklin Lakes, NJ). The ratio of PI-positive dead cells to all analyzed cells was measured to

evaluate complement-dependent cytotoxicity.

\section{$2.4 \quad$ CNSL model rats}

Eight-week old F344 (rnu/rnu) nude rats were purchased from CLEA Japan (Tokyo, Japan)

and were bred in Laboratory Animal Resource Center, University of Tsukuba. Experiments with rats described in this paper were approved by Laboratory Animal Resource Center, University of Tsukuba.

The nude rats were anesthetized with pentobarbital (Dainippon Sumitomo Pharma, Osaka, Japan) and immobilized with a brain stereotaxic apparatus. To create a port to administer RTX and serum, a 23-gauge stainless steel tube with a removable internal stopper was cannulated into the ipsilateral lateral ventricle at $1.6 \mathrm{~mm}$ lateral and $0.8 \mathrm{~mm}$ caudal to bregma, and fixed at a $4.5 \mathrm{~mm}$ depth from the cranial bone surface. The cannula was secured to the cranial surface with dental resin cement. Proper cannulation was confirmed in brain sections from rats sacrificed immediately after the administration of trypan blue through the cannula $(n=2)$. While the cannulation was performed, exponentially growing Raji cells were harvested at a density of $1 \times 10^{6}$ to $1.5 \times 10^{6}$ cells $/ \mathrm{mL}$ and washed once in phosphate-buffered saline without calcium and magnesium (PBS). After the cells were 
centrifuged and the supernatant was removed, the cell pellet was loosened by tapping, aspirated into a microinjector, and left on ice until inoculation. A $5-\mu \mathrm{L}$ aliquot of this pellet, which contained $1 \times 10^{6}$ to 3 $\times 10^{6}$ cells, was inoculated into the deep frontal lobe of the cerebrum of the above-prepared nude rats at $2 \mathrm{~mm}$ lateral and $3 \mathrm{~mm}$ rostral to bregma, and $4.5 \mathrm{~mm}$ deep to the cranial bone surface.

\subsection{Immunohistochemical analysis}

From days 9 to 11 after Raji cell inoculation, $100 \mu \mathrm{g}$ RTX or control immunoglobulin in $10 \mu \mathrm{L}$ of $0.154 \mathrm{M} \mathrm{NaCl}$ solution (saline) was administered with $10 \mu \mathrm{L}$ human serum once a day into the lateral ventricle through the cannula. The animals were euthanized and the brains were surgically removed 24 hours after the last administration, embedded in OCT compound (Sakura Finetechnical, Tokyo, Japan), frozen at $-80^{\circ} \mathrm{C}$, sliced at 3 or $6 \mu \mathrm{m}$ with a cryostat, and immunohistologically analyzed following a standard protocol. In brief, after blocking nonspecific adhesion with BPT solution (1\% bovine serum albumin [Sigma-Aldrich], PBS, and 0.1\% Tween 20 [Sigma-Aldrich]), the sections were incubated with antibody in BPT solution. To confirm the presence of the Raji-cell tumor in the brain, the sections were stained with RTX and Fluorescein isothiocyanate (FITC)-conjugated rabbit anti-human IgG immunoglobulin or control FITC-conjugated rabbit immunoglobulin (DAKO, Glostrup, Denmark).

To evaluate whether intraventricular RTX reached the tumor cells, consecutive sections were directly stained with FITC-conjugated rabbit anti-human IgG immunoglobulin or control 
FITC-conjugated rabbit immunoglobulin. Rats administered control immunoglobulin and human serum were stained in the same manner as negative controls. After rinses, the samples were covered with VECTASHIELD Mounting Medium with DAPI (Vector Laboratories, Burlingame, CA) and observed with an Axioplan2 fluorescent microscope (Carl Zeiss, Oberkochen, Germany). Images were taken and analyzed with an AxioCam CCD camera and AxioVision4.6 image processing software (Carl Zeiss), respectively.

\subsection{Treatment of lymphomas in CNSL model rats}

Once a day on day 5 through day 9 after Raji innoculation, CNSL model rats were intraventricularly administered with a mixture of $100 \mu \mathrm{g}(10 \mu \mathrm{L})$ of RTX or control immunoglobulin with $10 \mu \mathrm{L}$ of human serum, or a mixture of the same dose of RTX with saline. The survival data were collected from 10 independent experiments. The rats were observed and weighted once a day after intraventricular administration. When $>25 \%$ weight reduction or obvious weakness was observed, the rat was euthanized, and this time point was defined as the end of survival. The brains were removed from all of the rats, regardless of whether death was natural or by euthanasia. If no lymphoma was found in the brain, the animal was excluded from the analysis. Survival of each group was analyzed with the Kaplan-Meier method and log-rank test with StatCell version 2 software (OMS, Saitama, Japan), and 
1

2

3

4

5

7

8

9

10

11

12

13

14

15

16

17

18

19

20

21

22

23

24

25

26

27

28

29

30

31

32

33

34

35

36

37

38

39

40

41

42

43

44

45

46

47

48

49

50

51

52

53

54

55

56

57

58

59

60

61

62

63

64

65

significant differences were assumed at $P$ values $<0.05$. 
We first analyzed the induction of CDC by RTX and human serum in vitro using cells of the

CD20-positive human non-Hodgkin lymphoma B-cell line Raji as a target. Cytotoxicity was observed with the combination of RTX and serum, but not with the combinations of control immunoglobulin and serum or of RTX and heat-inactivated serum (Fig. 1). To investigate the efficacy of intraventricular RTX combined with serum, we generated a rat CNSL model in which Raji cells were inoculated into the deep white matter of the nude rat brain frontal lobe (Fig. 2). Tumor formation was confirmed histologically in brains removed 12 days after inoculation (Fig. 3). At the time of Raji cell inoculation, an indwelling cannula was implanted into the lateral ventricle on the ipsilateral side to use as the route of intraventricular administration (Fig. 2). Most rats recovered fully after the operation and those which did not recovered were excluded from the following experiments. Next, the rats were administered with RTX or control immunoglobulin and human serum via the cannula for three consecutive days beginning on day 9 after Raji inoculation. Rats recovered normally after general anesthesia and intraventricular administration of the study drugs. Twenty-four hours after the last administration, the brains were removed for analysis by immunostaining. We observed specific staining with an FITC-conjugated rabbit anti-human immunoglobulin (Fig. 4, upper panels), but not with a control FITC-conjugated rabbit immunoglobulin (middle panels); this staining was present only after the administration of RTX (Fig. 4, 
Exp. 1 and Exp. 2) and not after control human immunoglobulin [Fig. 4, Exp. 1 (control) and Exp. 2

(control). Note that control immunoglobulin was not detected even in the high sensitivity with some background in Exp. 1 (control) and Exp. 2 (control)]. The staining area largely overlapped with the CD20-positive Raji cell tumor area (Fig. 4, lower panels). This demonstrates that intraventricular RTX penetrated the ependymal layer and brain parenchyma, and finally reached and bound to lymphoma cells. To evaluate the anti-lymphoma effect of intraventricular RTX and serum, the tumor-inoculated rats were treated with either RTX plus serum, RTX plus saline, or control immunoglobulin plus serum. Lymphoma development was confirmed histologically in all the rats examined. A single rat in the RTX-plus-serum group died from technical error during the treatment period and was excluded from this analysis. The RTX-plus-serum group survived significantly longer than the control-immunoglobulin-plus-serum group $(P=0.049 ;$ Fig. 5). The RTX-plus-serum group also appeared to survive longer than the RTX-plus-saline group, although the difference was not statistically significant $(P=0.083)$. The survival times of the RTX-plus-saline and control-immunoglobulin-plus-serum groups did not differ from each other $(P=0.382)$. Body weights transiently decreased immediately after the intraventricular treatment in all arms, but all the rats subsequently re-gained the weight, which began to decrease shortly before the death (Supplemental Fig. $1)$. 


\section{Discussion}

A few rodent CNSL models have been described previously in which lymphoma cells were

directly inoculated into deep cerebral white matter $[25,26]$. On the basis of the information in these articles, we established a CNSL model in nude rats bearing a tumor made from the Raji cell line, which is widely used for CDC and ADCC assays with $\mathrm{RTX}$ in vitro [8, 10, 12] and in vivo [27, 28]. An intracerebral Raji cell tumor established in athymic mice was recently reported to recapitulate the histopathology and molecular features of primary CNSL [29]. Although the authors of that paper emphasized that their model was suitable for preclinical testing of novel agents, mice are too small and technically difficult to handle for many investigators. We therefore chose a nude rat as a host.

In this paper, we showed that intraventricular RTX reaches the tumor through the ependymal layer and brain parenchyma, although the fluorescence intensity of tumor-bound RTX was much weaker than the positive control staining (Fig. 4). This indicates that only a fraction of RTX penetrated the brain parenchyma, although the dilution associated with diffusion in the brain might also be attributed.

Despite the low concentration of RTX into the tumor, our results indicate that the binding of RTX to the Raji cell tumor induced tumor killing. Because this effect was dependent on human serum, it is likely that the cell killing was actually due to CDC against the Raji tumor cells in vivo. It is also possible that RTX and complement induced tumor cell phagocytosis by microglial cells, which are 
known to possess $\mathrm{Fc}$ and complement receptors [30, 31]. In addition, other effector cells such as monocytes and NK cells might also migrate to the tumor site after the initial CDC or phagocytosis. The relatively modest effect of intraventricular RTX and serum (Fig. 5) could be due to an insufficient dose of RTX at the tumor, given the low level of RTX binding to the tumor cells. Increasing the concentration of intraventricular RTX might therefore improve the efficacy in clinical settings. Alternatively, although clinical studies should be more complicated, directly administering monocytes or NK cells may be more advantageous to elicit continuous anti-lymphoma effect in the CNS.

Intrathecal or intraventricular RTX, administered as a single agent, has been reported to be effective against CNS lymphomas in clinical settings. The efficacy, however, has mainly been described for leptomeningeal involvement of B-cell lymphomas rather than the lesions in the brain parenchyma [20, 32, 33]. These reports are largely consistent with our findings that the survival times of the RTX-plus-saline and control-immunoglobulin-plus-serum groups did not differ from each other (Fig. 5).

Recently, a case of refractory primary CNSL that was treated with intraventricular RTX and autologous serum was reported [34]. The patient's condition initially deteriorated, but remarkable tumor regression occurred after 8 weeks. Our results provide experimental evidence in support of this clinical report. The authors of the clinical report discussed the possible induction of an adaptive immune response to explain the relatively slow clinical course, rather than the immediate effect expected from CDC. Because dendritic cells have been shown to emerge in the CNS in mouse CNS inflammation 
models [35], it is possible that, after CDC, CNSL-associated antigens were processed and presented on

brain dendritic cells and adaptive immunity was initiated, leading to tumor regression.

Our treatment model could be adapted for use in patients because intraventricular cannulation

and Ommaya reservoir implantation are routinely performed by neurosurgeons. Given the possible slow

effect, intraventricular RTX and autoserum treatment might be suitable as a consolidation or maintenance therapy for CNSL in clinical settings. Clinical trials are warranted. 


\section{Acknowledgments}

We thank Dr. J. Usui and Ms. K. Noguchi (University of Tsukuba) for technical assistance with

immunohistochemistry; Drs. T. Yamamoto, W. Tsuruta, and T. Tsurubuchi (University of Tsukuba) for technical advice with the rat surgical experiments and brain stereotaxic apparatus; and Drs. T. Sakurai (current affiliation, Kanazawa University) and T. Matsuki (current affiliation, University of Texas Southwestern Medical Center at Dallas) for technical assistance with the rat cannulation. This work was supported by the Aichi Cancer Research Foundation (Y. O.) and Research Grant of the Princess Takamatsu Cancer Research Fund (S. C.). 


\section{References}

1. Swerdlow SH, Campo E, Harris NL, Jaffe ES, Pileri SA, Stein H et al. WHO Classification of Tumours of Haematopoietic and Lymphoid Tissues, Fourth Edition. In. Lyon: International Agency for Research on Cancer (IARC), 2008.

2. Yamanaka R, Morii K, Shinbo Y, Homma J, Sano M, Tsuchiya N et al. Results of treatment of 112 cases of primary CNS lymphoma. Jpn J Clin Oncol. 2008; 38(5): 373-80.

3. Jahnke K, Thiel E. Treatment options for central nervous system lymphomas in immunocompetent patients. Expert Rev Neurother. 2009; 9(10): 1497-509.

4. Shimazu Y, Notohara K, Ueda Y. Diffuse large B-cell lymphoma with central nervous system relapse: prognosis and risk factors according to retrospective analysis from a single-center experience. Int J Hematol. 2009; 89(5): 577-83.

5. Stamenkovic I, Seed B. Analysis of two cDNA clones encoding the B lymphocyte antigen CD20 (B1, Bp35), a type III integral membrane protein. J Exp Med. 1988; 167(6): 1975-80.

6. Einfeld DA, Brown JP, Valentine MA, Clark EA, Ledbetter JA. Molecular cloning of the human B cell CD20 receptor predicts a hydrophobic protein with multiple transmembrane domains. Embo J. 1988; 7(3): 711-7.

7. Reff ME, Carner K, Chambers KS, Chinn PC, Leonard JE, Raab R et al. Depletion of B cells in vivo by a chimeric mouse human monoclonal antibody to CD20. Blood. 1994; 83(2): 435-45.

8. Flieger D, Renoth S, Beier I, Sauerbruch T, Schmidt-Wolf I. Mechanism of cytotoxicity induced by chimeric mouse human monoclonal antibody IDEC-C2B8 in CD20-expressing lymphoma cell lines. Cell Immunol. 2000; 204(1): 55-63.

9. Shimadoi S, Takami A, Kondo Y, Okumura H, Nakao S. Macrophage colony-stimulating factor enhances rituximab-dependent cellular cytotoxicity by monocytes. Cancer Sci. 2007; 98(9): 1368-72. 
10. van Meerten T, van Rijn RS, Hol S, Hagenbeek A, Ebeling SB. Complement-induced cell death by rituximab depends on CD20 expression level and acts complementary to antibody-dependent cellular cytotoxicity. Clin Cancer Res. 2006; 12(13): 4027-35.

11. Manches O, Lui G, Chaperot L, Gressin R, Molens JP, Jacob MC et al. In vitro mechanisms of action of rituximab on primary non-Hodgkin lymphomas. Blood. 2003; 101(3): 949-54.

12. Teeling JL, French RR, Cragg MS, van den Brakel J, Pluyter M, Huang $H$ et al. Characterization of new human CD20 monoclonal antibodies with potent cytolytic activity against non-Hodgkin lymphomas. Blood. 2004; 104(6): 1793-800.

13. Di Gaetano N, Cittera E, Nota R, Vecchi A, Grieco V, Scanziani E et al. Complement activation determines the therapeutic activity of rituximab in vivo. J Immunol. 2003; 171(3): 1581-7.

14. Golay J, Zaffaroni L, Vaccari T, Lazzari M, Borleri GM, Bernasconi S et al. Biologic response of B lymphoma cells to anti-CD20 monoclonal antibody rituximab in vitro: CD55 and CD59 regulate complement-mediated cell lysis. Blood. 2000; 95(12): 3900-8.

15. Cardarelli PM, Quinn M, Buckman D, Fang Y, Colcher D, King DJ et al. Binding to CD20 by anti-B1 antibody or $\mathrm{F}\left(\mathrm{ab}^{\prime}\right)(2)$ is sufficient for induction of apoptosis in B-cell lines. Cancer Immunol Immunother. 2002; 51(1): 15-24.

16. Byrd JC, Kitada S, Flinn IW, Aron JL, Pearson M, Lucas D et al. The mechanism of tumor cell clearance by rituximab in vivo in patients with B-cell chronic lymphocytic leukemia: evidence of caspase activation and apoptosis induction. Blood. 2002; 99(3): 1038-43.

17. Pedersen IM, Buhl AM, Klausen P, Geisler CH, Jurlander J. The chimeric anti-CD20 antibody rituximab induces apoptosis in B-cell chronic lymphocytic leukemia cells through a p38 mitogen activated protein-kinase-dependent mechanism. Blood. 2002; 99(4): 1314-9.

18. Harjunpaa A, Wiklund T, Collan J, Janes R, Rosenberg J, Lee D et al. Complement activation in circulation and central nervous system after rituximab (anti-CD20) treatment of B-cell lymphoma. Leuk Lymphoma. 2001; 42(4): 731-8.

19. Rubenstein JL, Combs D, Rosenberg J, Levy A, McDermott M, Damon L et al. Rituximab therapy for CNS lymphomas: targeting the leptomeningeal compartment. Blood. 2003; 101(2): 
466-8.

20. Schulz H, Pels H, Schmidt-Wolf I, Zeelen U, Germing U, Engert A. Intraventricular treatment of relapsed central nervous system lymphoma with the anti-CD20 antibody rituximab. Haematologica. 2004; 89(6): 753-4.

21. Johanson CE, Duncan JA, Stopa EG, Baird A. Enhanced prospects for drug delivery and brain targeting by the choroid plexus-CSF route. Pharm Res. 2005; 22(7): 1011-37.

22. Belichenko PV, Dickson PI, Passage M, Jungles S, Mobley WC, Kakkis ED. Penetration, diffusion, and uptake of recombinant human alpha-L-iduronidase after intraventricular injection into the rat brain. Mol Genet Metab. 2005; 86(1-2): 141-9.

23. Pardridge WM. Drug delivery to the brain. J Cereb Blood Flow Metab. 1997; 17(7): 713-31.

24. de Lange EC, de Boer BA, Breimer DD. Microdialysis for pharmacokinetic analysis of drug transport to the brain. Adv Drug Deliv Rev. 1999; 36(2-3): 211-227.

25. Saini M, Bellinzona M, Weichhold W, Samii M. A new xenograft model of primary central nervous system lymphoma. J Neurooncol. 1999; 43(2): 153-60.

26. Mineo JF, Scheffer A, Karkoutly C, Nouvel L, Kerdraon O, Trauet J et al. Using human CD20-transfected murine lymphomatous B cells to evaluate the efficacy of intravitreal and intracerebral rituximab injections in mice. Invest Ophthalmol Vis Sci. 2008; 49(11): 4738-45.

27. Hudson WA, Li Q, Le C, Kersey JH. Xenotransplantation of human lymphoid malignancies is optimized in mice with multiple immunologic defects. Leukemia. 1998; 12(12): 2029-33.

28. Griffiths GL, Mattes MJ, Stein R, Govindan SV, Horak ID, Hansen HJ et al. Cure of SCID mice bearing human B-lymphoma xenografts by an anti-CD74 antibody-anthracycline drug conjugate. Clin Cancer Res. 2003; 9(17): 6567-71.

29. Kadoch C, Dinca EB, Voicu R, Chen L, Nguyen D, Parikh S et al. Pathologic correlates of primary central nervous system lymphoma defined in an orthotopic xenograft model. Clin Cancer Res. 2009; 15(6): 1989-97. 
30. Carson MJ, Reilly CR, Sutcliffe JG, Lo D. Mature microglia resemble immature antigen-presenting cells. Glia. 1998; 22(1): 72-85.

31. Havenith CE, Askew D, Walker WS. Mouse resident microglia: isolation and characterization of immunoregulatory properties with naive CD4+ and CD8+ T-cells. Glia. 1998; 22(4): 348-59.

32. Rubenstein JL, Fridlyand J, Abrey L, Shen A, Karch J, Wang E et al. Phase I study of intraventricular administration of rituximab in patients with recurrent CNS and intraocular lymphoma. J Clin Oncol. 2007; 25(11): 1350-6.

33. Pradilla G, Wang PP, Gabikian P, Li K, Magee CA, Walter KA et al. Local intracerebral administration of Paclitaxel with the paclimer delivery system: toxicity study in a canine model. J Neurooncol. 2006; 76(2): 131-8.

34. Takami A, Hayashi T, Kita D, Nishimura R, Asakura H, Nakao S. Treatment of primary central nervous system lymphoma with induction of complement-dependent cytotoxicity by intraventricular administration of autologous-serum-supplemented rituximab. Cancer Sci. 2006; 97(1): 80-3.

35. Fischer HG, Reichmann G. Brain dendritic cells and macrophages/microglia in central nervous system inflammation. J Immunol. 2001; 166(4): 2717-26. 
Fig. 1

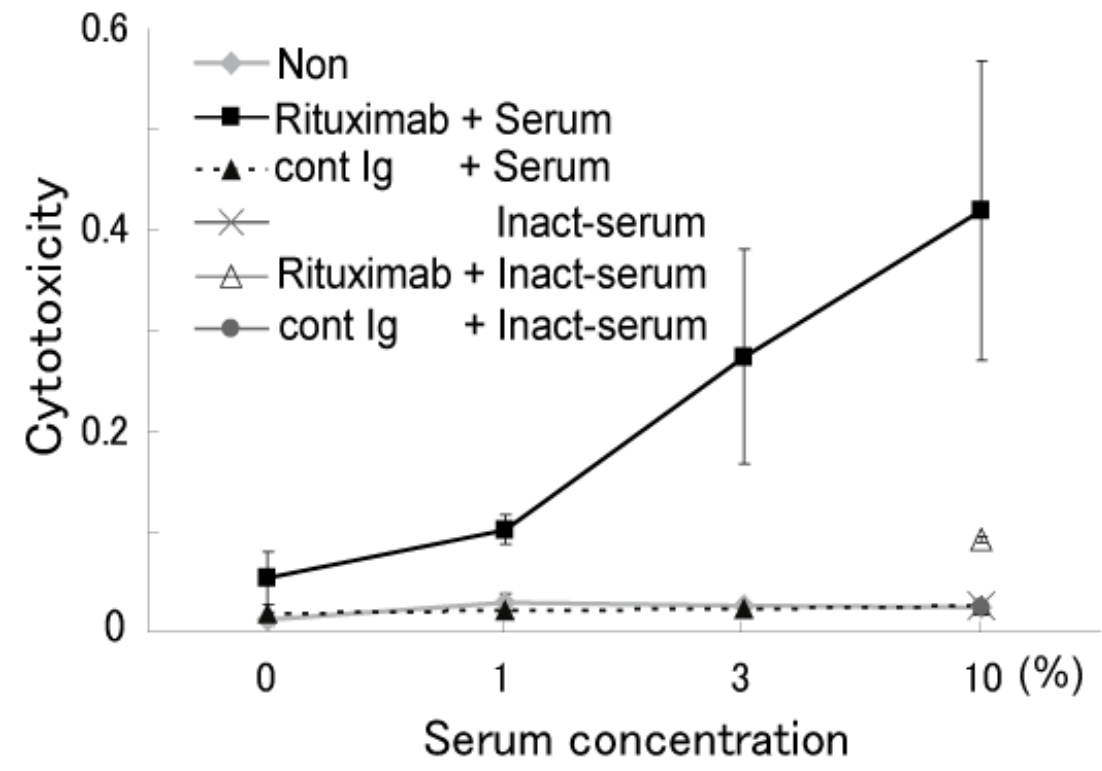

Complement-dependent cytotoxicity of rituximab and serum.

Complement-dependent cytotoxicity of Raji cells treated with the indicated concentrations of human serum in the presence of rituximab or control human immunoglobulin (cont Ig) was measured.

Inact-serum, heat-inactivated serum. Data are representative of two independent experiments. 
Fig. 2

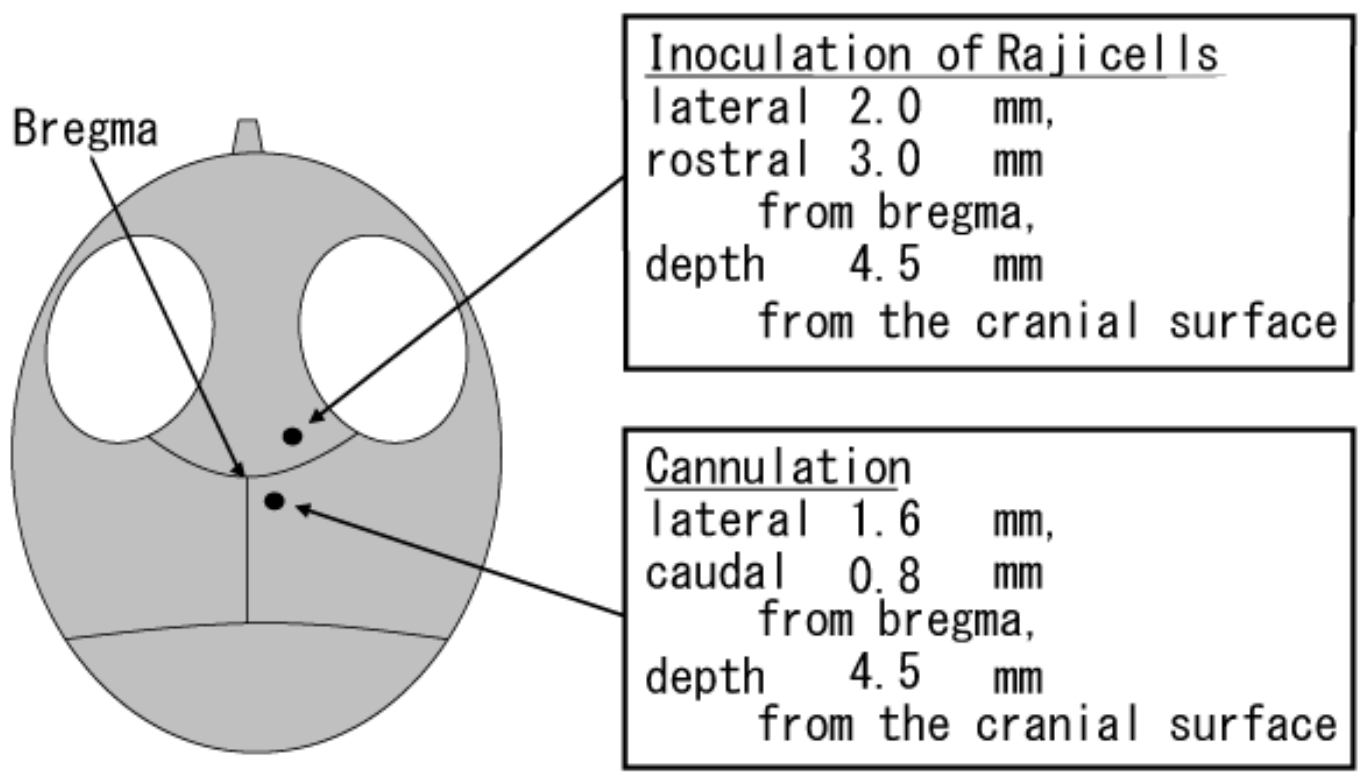

Positions of intracerebral inoculation of Raji cells and intraventricular cannulation.

Raji cells were inoculated into the deep frontal lobe. The cannula was placed in the ipsilateral lateral ventricle. 
Fig. 3

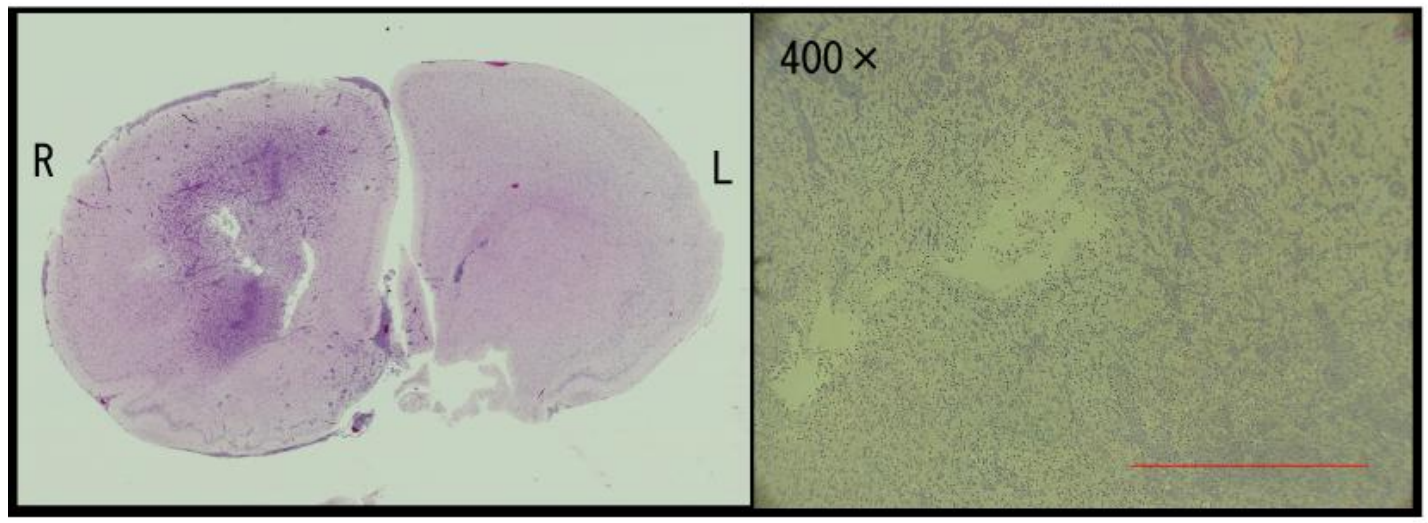

Histological study of rat cerebrum after Raji cell inoculation.

A coronal section of nude rat cerebrum was stained with hematoxylin and eosin 12 days after

Raji cell inoculation. Left panel: macroscopic finding. The Raji cell tumor is depicted by basophilic staining in the right hemisphere. Right panel: histology in a high-power field (400×). Raji cells diffusely infiltrated in the deep frontal lobe of the cerebrum. The scale bar indicates $1 \mathrm{~mm}$. 
Fig. 4
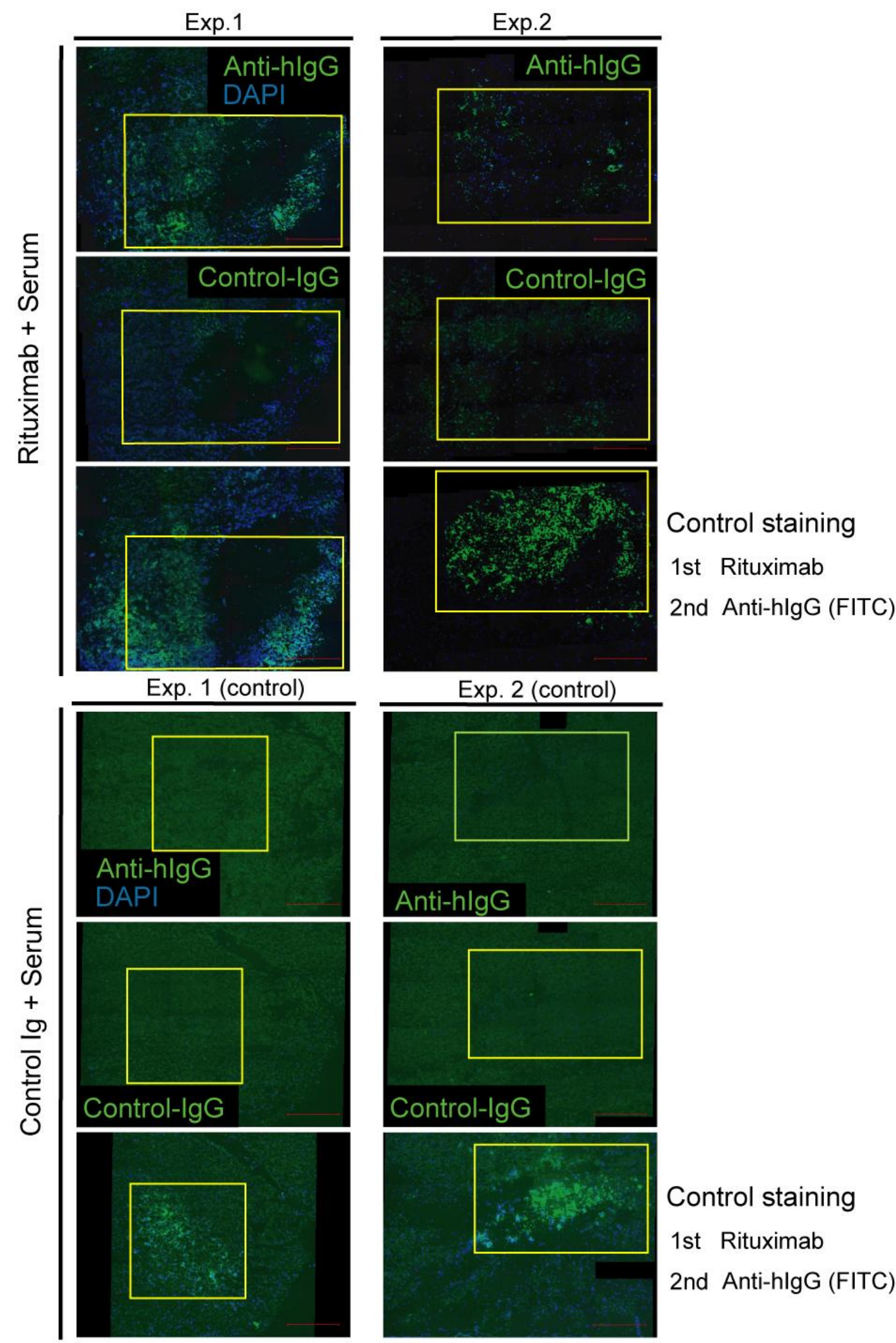

Control staining

1st Rituximab

2nd Anti-hlgG (FITC) 
Specific binding of rituximab to tumor cells in the cerebrum after intraventricular administration.

Rituximab plus serum (Exp. 1 and Exp. 2) or control immunoglobulin plus serum [Exp. 1 (control) and Exp. 2 (control)] was administered into the lateral ventricles of the rats from day 9 to day 11 after Raji cell inoculation. The animals were euthanized, and the brains were surgically removed and stained 24 hours after the last administration. Sections were stained with FITC-conjugated anti-human IgG (Anti-hIgG, upper panels) or FITC-conjugated control immunoglobulin (middle panels). To clarify the margin of the Raji cell tumor, the section was stained with rituximab as the primary antibody and FITC-conjugated anti-human IgG as a secondary antibody (lower panels). DAPI (4', 6-diamidino-2-phenylindole) was used for nuclear staining. The yellow box depicts the area of the Raji cell infiltration and the scale bar indicates $500 \mu \mathrm{m}$. Data are representative of three independent experiments. 
Fig. 5

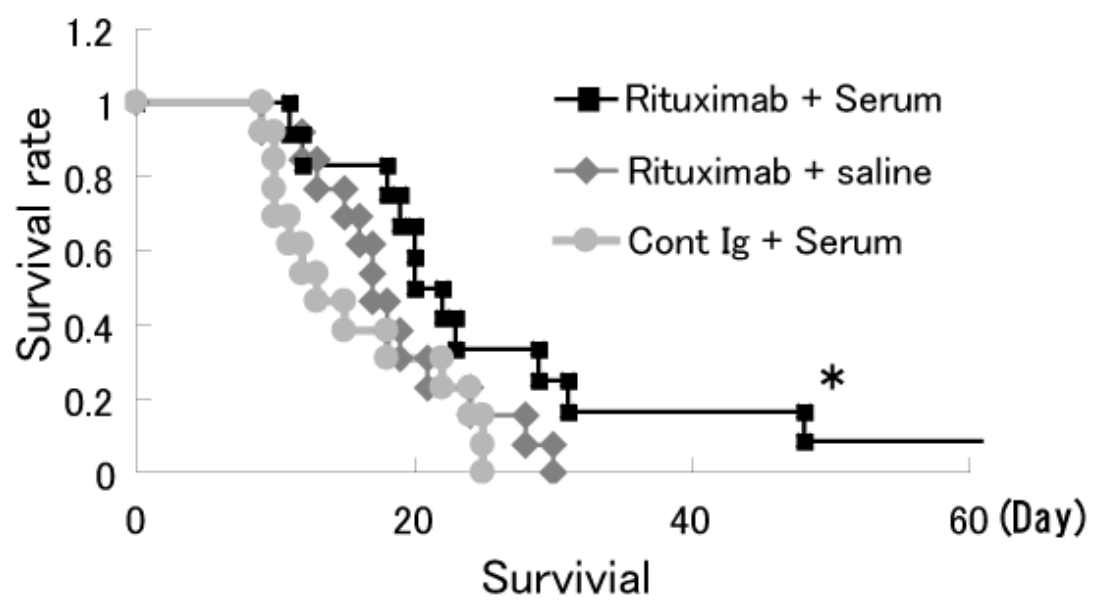

Therapeutic effect of intraventricular administration of rituximab and serum.

Kaplan-Meier survival curves. Data were collected from 10 pooled experiments. The rats were treated with rituximab plus serum (Rituximab + Serum, $n=12)$, rituximab plus saline (Rituximab + saline, $\mathrm{n}=13$ ), or control immunoglobulin plus serum (Cont Ig + Serum, $n=13$ ). The asterisk indicates a statistically significant difference between rituximab plus serum and control immunoglobulin plus serum $(P=0.049)$ in a log-rank test. 
Supplementary Material
Click here to download Supplementary Material: Supplemental Fig1.docx

Supplementary Material
Click here to download Supplementary Material: Supplemental Fig1.docx

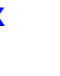

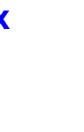

upplementary Material: Supplemental Fig1.docx

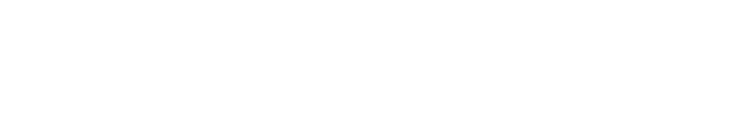

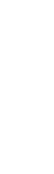
(1) (1) (1)

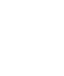
(1)

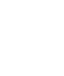
(1) (1) (1) (1) . . . . . . .

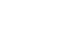
. 